bioRxiv preprint doi: https://doi.org/10.1101/004481; this version posted July 21, 2014. The copyright holder for this preprint (which was

\title{
Predicting evolutionary site variability from structure in viral proteins: buriedness, packing, flexibility, and design
}

\author{
Amir Shahmoradi · Dariya K. Sydykova · \\ Stephanie J. Spielman • Eleisha L. Jackson • \\ Eric T. Dawson • Austin G. Meyer • Claus O. Wilke
}

\begin{abstract}
Several recent works have shown that protein structure can predict site-specific evolutionary sequence variation. In particular, sites that are buried and/or have many contacts with other sites in a structure have been shown to evolve more slowly, on average, than surface sites with few contacts. Here, we present a comprehensive study of the extent to which numerous structural properties can predict sequence variation. The quantities we considered include buriedness (as measured by relative solvent accessibility), packing density (as measured by contact number), structural flexibility (as measured by B factors, root-mean-square fluctuations, and variation in dihedral angles), and variability in designed structures. We obtained structural flexibility measures both from molecular dynamics simulations performed on 9 non-homologous viral protein structures and from variation in homologous variants of those proteins, where available. We obtained measures of variability in designed structures from flexible-backbone design in the Rosetta software. We found that most of the structural properties correlate with site variation in the majority of structures, though the correlations are generally weak (correlation coefficients of 0.1 to 0.4 ). Moreover, we found that buriedness and packing density were better predictors of evolutionary variation than was structural flexibility. Finally, variability in designed structures was a weaker predictor of evolutionary variability than was buriedness or packing density, but it was comparable in its predictive power to the best structural flexibility measures. We conclude that simple measures of buriedness and packing density are better predictors of evolutionary variation than are more complicated predictors obtained from dynamic simulations, ensembles of homologous structures, or computational protein design.
\end{abstract}

\section{Introduction}

Patterns of amino-acid sequence variation in protein-coding genes are shaped by the structure and function of the expressed proteins (Wilke and Drummond, 2010, Liberles et al, 2012, Marsh and Teichmann. 2014). As the most basic reflection of this relationship, buried residues in proteins tend to be more evolutionarily conserved than exposed residues (Overington et al, 1992, Goldman et al, 1998, Mirny and Shakhnovich, 1999, Dean et al, 2002). More specifically, when evolutionary variation is plotted as a function of Relative Solvent Accessibility (RSA, a measure of residue buriedness), the relationship falls, on average, onto a straight line with a positive slope (Franzosa and Xia, 2009, Ramsey et al, 2011; Franzosa and Xia, 2012, Scherrer et al, 2012). Importantly, however, this relationship represents an average over many sites and many proteins. At the level of individual sites in individual proteins, RSA is often only weakly correlated with evolutionary variation (Meyer and Wilke, 2013; Meyer et al, 2013, Yeh et al, 2014b).

Other structural measures, such as residue contact number $(\mathrm{CN})$, have also been shown to correlate with sequence variability (Liao et al, 2005, Franzosa and Xia, 2009, Yeh et al, 2014b), and some have argued that CN predicts evolutionary variation better than RSA (Yeh et al, 2014b a). Because CN may

Amir Shahmoradi

Department of Physics, The University of Texas at Austin, TX, 78712

Amir Shahmoradi · Dariya K. Sydykova · Stephanie J. Spielman · Eleisha L. Jackson · Eric T. Dawson · Austin G. Meyer · Claus O. Wilke

Department of Integrative Biology, Center for Computational Biology and Bioinformatics, and Institute for Cellular and Molecular Biology, The University of Texas at Austin, TX, 78712. E-mail: wilke@austin.utexas.edu 
bioRxiv preprint doi: https://doi.org/10.1101/004481; this version posted July 21, 2014. The copyright holder for this preprint (which was

be a proxy for residue and site-specific backbone flexibility (Halle, 2002), a positive trend between local structural variability and sequence variability may also exist (Yeh et al, 2014b). Indeed, several authors have suggested that such protein dynamics may play a role in sequence variability (Liu and Bahar, 2012 . Nevin Gerek et al, 2013, Marsh and Teichmann, 2014). However, a recent paper argued against the flexibility model, on the grounds that evolutionary rate is not linearly related to flexibility (Huang et al, 2014).

While RSA and CN can be calculated in a straightforward manner from individual crystal structures, measures of structural flexibility, either at the side-chain or the backbone level, are more difficult to obtain. Two viable approaches to measuring structural flexibility are (i) examining existing structural data or (ii) simulating protein dynamics. NMR ensembles may approximate physiologically relevant structural fluctuations. Similar fluctuations are observed in ensembles of homologous crystal structures (Maguida et al, 2008, Echave and Fernández, 2010). The thermal motion of atoms in a crystal are recorded in B factors, which are available for every atom in every crystal structure. To measure protein fluctuations using a simulation approach, one can either use coarse-grained modeling, e.g. via Elastic Network Models (Sanejouand, 2013), or atom-level modeling, e.g. via molecular dynamics (MD) (Karplus and McCammon, 2002). However, it is not well understood which, if any, of these measures of structural flexibility provide insight into the evolutionary process, in particular into residue-specific evolutionary variation.

Here, we provide a comprehensive analysis of the extent to which numerous different structural quantities predict evolutionary sequence (amino-acid) variation. We considered two measures of evolutionary sequence variation: site entropy, as calculated from homologous protein alignments, and evolutionary rate. As structural predictors, we included buriedness (RSA), packing density (CN), and measures of structural flexibility, including B factors, several measures of backbone and side-chain variability obtained from MD simulations, and backbone variability obtained from alignments of homologous crystal structures. We additionally considered site variability, as predicted from computational protein design with Rosetta.

On a set of nine viral proteins, RSA and CN generally performed better at predicting evolutionary site variation than did either measures of structural flexibility or computational protein design. Among the measures of structural flexibility, measures of side-chain variability performed better than do measures of backbone variability, possibly because the former are more tightly correlated with residue packing. Finally, site variability predicted from computational protein design performed worse than the bestperforming measures of structural fluctuations.

\section{Materials and Methods}

Sequence data, alignments, and evolutionary rates

All viral sequences except influenza sequences were retrieved from http://hfv.lanl.gov/components/ sequence/HCV/search/searchi.html. The sequences were truncated to the desired genomic region but not in any other way restricted. Influenza sequences were downloaded from http://www.fludb.org/ brc/home.spg?decorator=influenza. We only considered human influenza A, H1N1, excluding H1N1 sequences derived from the 2009 Swine Flu outbreak or any sequence from before 1998, but we did not place any geographic restrictions.

For all viral sequences, we removed any sequence that was not in reading frame, any sequence which was shorter than $80 \%$ of the longest sequence for a given viral protein (so as to remove all partial sequences), and any sequence containing any ambiguous characters. Alignments were constructed using amino-acid sequences with MAFFT (Katoh et al, 2002, 2005), specifying the --auto flag to select the optimal algorithm for the given data set, and then back-translated to a codon alignment using the original nucleotide sequence data.

To assess site-specific sequence variability in amino-acid alignments, we calculated the Shannon entropy $\left(H_{i}\right)$ at each alignment column $i$ :

$$
H_{i}=-\sum_{j} P_{i j} \ln P_{i j}
$$

where $P_{i j}$ is relative frequency of amino acid $j$ at position $i$ in the alignment.

For each alignment, we also calculated evolutionary rates, as described (Spielman and Wilke, 2013). In brief, we generated a phylogeny for each codon alignment in RAxML (Stamatakis, 2006) using the 
bioRxiv preprint doi: https://doi.org/10.1101/004481; this version posted July 21, 2014. The copyright holder for this preprint (which was

GTRGAMMA model. Using the codon alignment and phylogeny, we inferred evolutionary rates with a Random Effects Likelihood (REL) model, using the HyPhy software (Kosakovsky Pond et al, 2005). The REL model was a variant of the GY94 evolutionary model (Goldman and Yang, 1994) with five $\omega$ rate categories as free parameters. We employed an Empirical Bayes approach (Yang, 2000) to infer $\omega$ values for each position in the alignment. These $\omega$ values represent the evolutionary-rate ratio $d N / d S$ at each site.

Protein crystal structures

A total of 9 viral protein structures were selected for analysis, as tabulated in Table 1 . Sites in the PDB structures were mapped to sites in the viral sequence alignments via a custom-built python script that creates a consensus map between a PDB sequence and all sequences in an alignment.

For each of the viral proteins, homologous structures were identified using the blast.pdb function of the R package Bio3D (Grant et al, 2006). BLAST hits were retained if they had $\geq 35 \%$ sequence identity and $\geq 90 \%$ alignment length. Among the retained hits, we subsequently identified sets of homologous structures with unique sequences and with mutual pairwise sequence divergences of $\geq 2 \%, \geq 5 \%$, and $\geq 10 \%$.

Molecular Dynamics Simulations

Molecular dynamics (MD) simulations were carried out using the GPU implementation of the Amber12 simulation package (Salomon-Ferrer et al, 2013) with the most recent release of the Amber fixed-charge force field (ff12SB; c.f., AmberTools13 Manual). Prior to MD production runs, all PDB structures were first solvated in a box of TIP3P water molecules (Jorgensen et al, 1983) such that the structures were at least $10 \AA$ away from the box walls. Each individual system was then energy minimized using the steepest descent method for 1000 steps, followed by conjugate gradient for another 1000 steps. Then, the structures were constantly heated from $0 \mathrm{~K}$ to $300 \mathrm{~K}$ for $0.1 \mathrm{~ns}$, followed by $0.1 \mathrm{~ns}$ constant pressure simulations with positional harmonic restraints on all atoms to avoid instabilities during the equilibration process. The systems were then equilibrated for another 5 ns without positional restraints, each followed by $15 \mathrm{~ns}$ of production simulations for subsequent post-processing and analyses. All equilibration and production simulations were run using the SHAKE algorithm (Ryckaert et al, 1977). Langevin dynamics were used for temperature control.

Measures of buriedness, packing density, and structural flexibility

As a measure of residue buriedness, we calculated Relative Solvent Accessibility (RSA). To calculate RSA, we first calculated the Accessible Surface Area (ASA) for each residue in each protein, using the DSSP software (Kabsch and Sander, 1983). We then normalized ASA values by the theoretical maximum ASA of each residue (Tien et al, 2013) to obtain RSA. We considered two measures of local packing density, contact number $(\mathrm{CN})$ and weighted contact number $(\mathrm{WCN})$. We calculated $\mathrm{CN}$ for each residue as the total number of $\mathrm{C} \alpha$ atoms surrounding the $\mathrm{C} \alpha$ atom of the focal residue within a spherical neighborhood of a predefined radius $r_{0}$. Following Yeh et al $(2014 \mathrm{~b})$, we used $r_{0}=13 \AA$. We calculated WCN as the total number of surrounding $\mathrm{C} \alpha$ atoms for each focal residue, weighted by the inverse square separation between the $\mathrm{C} \alpha$ atoms of the focal residue and the contacting residue, respectively (Shih et al, 2012).

In most analyses, we actually used the inverse of $\mathrm{CN}$ and/or $\mathrm{WCN}, \mathrm{iCN}=1 / \mathrm{CN}$ and $\mathrm{iWCN}=$ 1/WCN. Note that for Spearman correlations, which we use throughout here, replacing a variable by its inverse changes the sign of the correlation coefficient but not the magnitude.

As measures of structural flexibility, we considered RMSF, variability in backbone and side-chain dihedral angles, and B factors. We calculated RMSF for $\mathrm{C} \alpha$ atoms based on both MD trajectories and homologous crystal structures. For MD trajectories, we calculated RMSF as

$$
\operatorname{RMSF}_{j}=\left[\sum_{i}\left(\mathbf{r}_{i}^{(j)}-\mathbf{r}_{0}^{(j)}\right)^{2}\right]^{1 / 2}
$$

where $\mathrm{RMSF}_{j}$ is the root-mean-square fluctuation at site $j, \mathbf{r}_{i}^{(j)}$ is the position of the C $\alpha$ atom of residue $j$ at MD frame $i$, and $\mathbf{r}_{0}^{(j)}$ is the position of the $\mathrm{C} \alpha$ atom of residue $j$ in the original crystal structure. 
bioRxiv preprint doi: https://doi.org/10.1101/004481; this version posted July 21, 2014. The copyright holder for this preprint (which was

To calculate RMSF from homologous structures, we first aligned the structures using the Bio3D package (Grant et al, 2006), and then we calculated

$$
\operatorname{RMSF}_{j}=\left[\sum_{i} w_{i}\left(\mathbf{r}_{i}^{(j)}-\left\langle\mathbf{r}^{(j)}\right\rangle\right)^{2}\right]^{1 / 2},
$$

where $\mathbf{r}_{i}^{(j)}$ now stands for the position of the C $\alpha$ atom of residue $j$ in structure $i,\left\langle\mathbf{r}^{(j)}\right\rangle$ is the mean position of that $\mathrm{C} \alpha$ atom over all aligned structures, and $w_{i}$ is a weight to correct for potential phylogenetic relationship among the aligned structures. The weights $w_{i}$ were calculated using BranchManager (Stone and Sidow, 2007), based on phylogenies built with RAxML as before.

To assess variability in backbone and side-chain dihedral angles, we calculated $\operatorname{Var}(\phi), \operatorname{Var}(\psi)$, and $\operatorname{Var}\left(\chi_{1}\right)$. The variance of a dihedral angle was defined according to the most common definition in directional statistics: First, a unit vector $\mathbf{x}_{i}$ is assigned to each dihedral angle $\alpha_{i}$ in the sample. The unit vector is defined as $\mathbf{x}_{i}=\left(\cos \left(\alpha_{i}\right), \sin \left(\alpha_{i}\right)\right)$. The variance of the dihedral angle is then defined as

$$
\operatorname{Var}(\alpha)=1-\|\langle\mathbf{x}\rangle\|,
$$

where $\|\langle\mathbf{x}\rangle\|$ represents the length of the mean $\langle\mathbf{x}\rangle$, calculated as $\langle\mathbf{x}\rangle=\sum_{i} \mathbf{x}_{i} / n$. Here, $n$ is the sample size. The variance of a dihedral angle is, by definition, a real number in the range $[0,1]$, with $\operatorname{Var}(\alpha)=0$ corresponding to the minimum variability of the dihedral angle and $\operatorname{Var}(\alpha)=1$ to the maximum, respectively (Berens, 2009). Since the $\chi_{1}$ angle is undefined for Ala and Gly we excluded all sites with these residues in analyses involving $\chi_{1}$.

$\mathrm{B}$ factors were extracted from the crystal structures. We only considered the B factors of the C $\alpha$ atom of each residue.

\section{Sequence Entropy from Designed Proteins}

Designed entropy was calculated as described (Jackson et al, 2013). In brief, proteins were designed using RosettaDesign (Version 39284) (Leaver-Fay et al, 2011) using a flexible backbone approach. This was done for all PDB structures in Table 1 as initial template structures. For each template, we created a backbone ensemble using the Backrub method (Smith and Kortemme, 2008). The temperature parameter in Backrub was set to 0.6, allowing for an intermediate amount of flexibility. We had previously found in a different data set that intermediate flexibility gave the highest congruence between designed and observed site variability (Jackson et al, 2013).

For each of the 9 template structures we designed 500 proteins.

Availability of data and methods

All details of simulations, input/output files, and scripts for subsequent analyses are available to view or download at https://github.com/clauswilke/structural_prediction_of_ER

\section{Results}

Data set and structural variables considered

Our goal in this work was to determine which structural properties best predict amino-acid variability at individual sites in viral proteins. To this end, we selected 9 viral proteins for which we had both highquality crystal structures and abundant sequences to assess evolutionary sequence variation (Table 1). We quantified evolutionary variability in two ways: by calculating sequence entropies for each alignment column, and by calculating site-specific evolutionary-rate ratios $\omega=d N / d S$ (see Methods for details). Throughout this paper, we primarily report results obtained for sequence entropy. Results for $\omega$ were largely comparable, with some specific caveats detailed below.

As predictors of evolutionary variability, we considered buriedness, packing density, and residue flexibility. We additionally considered the variation seen in computationally designed protein variants. Buriedness quantifies the extent to which a residue is protected from solvent. We determined residue buriedness by calculating the relative solvent accessibility (RSA), which represents the relative proportion of a residue's surface in contact with solvent. 
bioRxiv preprint doi: https://doi.org/10.1101/004481; this version posted July 21, 2014. The copyright holder for this preprint (which was

Packing density quantifies how many other residues a given residue interacts with. We determined packing density by calculating contact number $(\mathrm{CN})$ and weighted contact number (WCN). CN counts the number of contacts within a sphere of a given radius around the $\alpha$-carbon of the focal residue, while WCN weights contacts by the distance between the two residues. Residue buriedness and packing density tend to be (anti-)correlated but measure qualitatively different properties of a residue. In particular, in the core of a protein, buriedness is always zero but packing density can vary. Because contact numbers decline as relative solvent accessibility increases, we replaced $\mathrm{CN}$ and $\mathrm{WCN}$ with their inverses, $\mathrm{iCN}=1 / \mathrm{CN}$ and $\mathrm{iWCN}=1 / \mathrm{WCN}$, in most analyses. Importantly, as Spearman rank correlations were used, this substitution only changed the sign of correlations but not the magnitude.

Measures of structural flexibility assess the extent to which a residue fluctuates in space as a protein undergoes thermodynamic fluctuations in solution. We quantified these fluctuations using several different measures. We considered B factors, which measure the spatial localization of individual atoms in a protein crystal, RMSF, the root mean-square fluctuation of the $\mathrm{C} \alpha$ atom over time, and variability in side-chain and backbone dihedral angles, including $\operatorname{Var}\left(\chi_{1}\right), \operatorname{Var}(\phi)$, and $\operatorname{Var}(\psi)$. We employed two broad approaches, one using PDB crystal structures and one using molecular dynamics (MD) simulations, to obtain these measurements. Crystal structures yielded measures for B factors and RMSF; we obtained B factors from individual protein crystal structures, given in Table 1, and we calculated RMSF from aligned homologous crystal structures for those proteins which had sufficient sequence variation among crystal structures (see Methods and Table 2 for details). MD simulations yielded measures for RMSF and variability in residue dihedral and side-chain angles. More specifically, we simulated MD trajectories for all crystal structures in Table 1. For each protein, we equilibrated the structure, simulated 15ns of chemical time, and recorded snapshots of the simulated structure every 10ps (see Methods for details). We obtained RMSF and angle variabilities from these snapshots. Additionally, we calculated time-averaged values of RSA, CN, and WCN. We also refer to these time-averaged measures as MD RSA, MD CN, and MD WCN, respectively. Unless specified otherwise, all results reported below were obtained using MD RSA, MD CN, and MD WCN.

As an alternative to predicting evolutionary variation from simple structural measures such as contact density or backbone flexibility, one can also predict evolutionary variation via a protein-design approach (Dokholyan and Shakhnovich, 2001, Ollikainen and Kortemme, 2013, Jackson et al, 2013). In this case, one takes the protein structure of interest, replaces all residue side chains with randomly-chosen alternatives, and uses a coarse-grained or atom-level energy function to assess which side-chain choices are consistent with the backbone conformation of the focal structure. We have recently used this approach to compare natural and designed sequence variability in cellular proteins (Jackson et al, 2013), and we have found that (i) flexible-backbone design, where small backbone movements are allowed during the design phase, outperformed fixed-backbone design, and (ii) intermediate backbone flexibility, obtained via an intermediate design temperature, produced the highest congruence between designed and natural sequences. Similarly, Dokholyan and Shakhnovich (2001) had previously found that an intermediate temperature parameter gave the best agreement between designed and natural sequences in their model. Inspired by these prior results, we investigated here how protein design performed relative to simpler structural quantities. For all proteins in our study (Table 1), we used the Rosetta protein-design platform (Leaver-Fay et al, 2011) to generate 500 designed variants. We then calculated the sequence entropy at each alignment position of the designed variants. We refer to the resulting quantity as the designed entropy. We chose a design temperature of $T=0.6$, which was near the optimal range in our previous work (Jackson et al, 2013).

\section{Evaluating structural predictors of evolutionary sequence variation}

We began by comparing the Spearman correlations of sequence entropy with six different measures of local structural flexibility: B factors, RMSF obtained from MD simulations (MD RMSF), and RMSF obtained from crystal structures (CS RMSF), and variability in backbone and side-chain dihedral angles $(\phi, \psi$, and $\left.\chi_{1}\right)$. The correlation strengths of these quantities with entropy are shown in Figure 1 . Significant correlations $(P<0.05)$ are shown with filled symbols, and non-significant correlations are shown with empty symbols $(P \geq 0.05)$. We found that the variability in backbone dihedral angles, $\operatorname{Var}(\phi)$ and $\operatorname{Var}(\psi)$, explained the least variation in sequence entropy, while the variability in the side-chain dihedral angle, $\operatorname{Var}\left(\chi_{1}\right)$, explained, on average, more variation in sequence entropy than did any other measure of structural flexibility. B factors and the two measures of RMSF explained, on average, approximately the same amount of variation in entropy, even though the results for individual proteins were somewhat discordant (see also next sub-section). 
bioRxiv preprint doi: https://doi.org/10.1101/004481; this version posted July 21, 2014. The copyright holder for this preprint (which was

Based on results from the above analysis, we proceeded to compare the relative explanatory power among the best-performing measures of structural flexibility ( $\operatorname{Var}\left(\chi_{1}\right)$, MD RMSF, and B factors) with buriedness (RSA), packing density (iWCN), and designed entropy. Figure 2 shows the Spearman correlation coefficients between sequence entropy and each of the aforementioned quantities, for all proteins in our analysis. In this figure, several patterns emerged. First, nearly all correlations were positive and most were statistically significant, with the main exception of the Marburg virus RNA binding domain (PDB ID 4GHA). This protein only showed a single significant negative correlation between sequence entropy and $\operatorname{Var}\left(\chi_{1}\right)$. Second, correlations were generally weak, such that no correlation coefficient exceeded 0.4. Third, on average, correlations were strongest for RSA and iWCN, yielding average correlations of $\rho=0.23$ and $\rho=0.22$, respectively. Fourth, designed entropy performed worse than RSA or iWCN as a predictor of evolutionary sequence variability, but it performed roughly the same as the three flexibility measures in this figure; the values of designed entropy, $\operatorname{Var}\left(\chi_{1}\right), \mathrm{MD}$ RMSF, and B factors showed average correlations of $\rho=0.13, \rho=0.14, \rho=0.11$, and $\rho=0.12$, respectively.

\section{MD time-averages vs. crystal-structure snapshots}

Except for analyses involving B factors and CS RMSF, we obtained structural measures by averaging quantities over MD trajectories. This approach, however, did not reflect conventional practice for measuring RSA, CN, or WCN, which are typically measured from individual crystal structures. Therefore, we examined whether MD time-averages differed in any meaningful way from estimates obtained from crystal structures, and whether these estimates differed in their predictive power for evolutionary sequence variation.

As shown in Table 3, RSA, CN, and WCN from crystal structures were highly correlated with their corresponding MD trajectory time-averages, for all protein structures we examined (Spearman correlation coefficients of $>0.9$ in all cases). Furthermore, the correlation coefficients we obtained when comparing the crystal-structure based measures to sequence entropy were virtually identical to coefficients obtained from the MC trajectory correlations (Figure $3 \mathrm{~A}-\mathrm{C}$ ). Thus, in terms of predicting evolutionary variation, RSA, CN, and WCN values obtained from static structures performed as well as their MD equivalents averaged over short time scales.

By contrast, correlations between corresponding MD RMSF to CS RMSF measures were sometimes quite different, with correlation coefficients ranging from 0.218 to 0.723 (Table 3). Consequently, for the two proteins for which MD RMSF was the least correlated with CS RMSF (hepatitis C protease and Rift Valley fever nucleoprotein), the strength of correlation between site entropy and RMSF depended substantially on how RMSF was calculated (Figures 1 and 3D).

Finally, we examined whether correlations between sequence entropy and B factors or the two RMSF measures were comparable (Figure 4). Again, we found that correlations between sequence entropy and $\mathrm{B}$ factors were generally different from those obtained for both MD RMSF and CS RMSF. This result highlighted that, while B factors, MD RSMF, and CS RMSF all measure backbone flexibility, they each contain distinct information about evolutionary sequence variability in our data set.

\section{Sequence entropy vs. evolutionary-rate ratio $\omega$}

In the previous subsections, we used sequence entropy as a measure of site-wise evolutionary variation. While sequence entropy is a simple and straightforward measure of site variability, it has two potential drawbacks. First, while measured from homologous protein alignments, sequence entropy doesn't correct for the phylogenetic relationship of those alignment sequences. Hence, entropy can be biased if some parts of the phylogeny are more densely sampled than others. Second, entropy does not take the actual substitution process into account. As a result, a single substitution near the root of the tree can result in a comparable entropy to a sequence of substitutions toggling back and forth between two amino acids.

To consider an alternative quantity of evolutionary variation that doesn't suffer from either of these drawbacks, we calculated the evolutionary-rate ratio $\omega=d N / d S$ for all proteins at all sites, and repeated all analyses with $\omega$ instead of entropy. We found that results generally carried over, but with somewhat weaker correlations. Figure 5 plots, for each protein, the Spearman correlations between $\omega$ and our various predictors versus the correlation between entropy and our predictors. Most data points fall below the $x=y$ line and are shifted downwards by approximately 0.1 . Thus, correlations of structural quantities and designed entropy with $\omega$ are, on average, approximately 0.1 smaller than correlations of the same quantities with sequence entropy. 
bioRxiv preprint doi: https://doi.org/10.1101/004481; this version posted July 21, 2014. The copyright holder for this preprint (which was

Multi-variate analysis of structural predictors

The various structural quantities we have considered are by no means independent of each other. Measures of buriedness and packing density co-vary with each other, as do measures of structural flexibility. Further, the latter co-vary with the former, as does designed entropy. Therefore, we conducted a joint multivariate analysis, which included most structural quantities considered in this work. We employed this strategy to determine the extent to which these quantities contained independent information about sequence variability while additionally assessing whether combining multiple structural quantities yielded improved predictive power. We employed a principal component (PC) regression approach, which has previously been used successfully to disentangle genomic predictors of whole-protein evolutionary rates (Drummond et al, 2006, Bloom et al, 2006). For each analysis described below, we first carried out a PC analysis of the predictor variables (i.e., the structural quantities such as RSA and RMSF), and we subsequently regressed the response (either sequence entropy or $\omega$ ) against the individual components. Note that variables were not rank-transformed for this analysis.

For a first PC analysis, we pooled all structural quantities and then regressed entropy against each PC separately, for all proteins in our data set. This strategy allowed us to analyze all proteins in our data set individually but in such a way that results were comparable from one protein to the next. We excluded CS RMSF from this analysis, so that we could include results from all nine viral proteins. The results of this analysis are shown in Figure 6. The first component (PC1) explained, on average, the largest amount of variation in sequence entropy (see Figure 6A). PC3 yielded the second-highest $r^{2}$ value, on average, while all other components explained very little variation in sequence entropy. When looking at the composition of the components, we found that RSA, iWCN, RMSF, and $\operatorname{Var}\left(\chi_{1}\right)$ all loaded strongly on PC1, while PC2 and PC3 where primarily represented by designed entropy and B factors (see Figure 6B and C). RMSF also had moderate loadings on PC3. Interestingly, designed entropy and B factors load with equal signs on PC2 but with opposite signs on PC3.

We interpreted PC1 to represent a buriedness/packing-density component. By definition, PC1 measures the largest amount of variation among the structural quantities, and all structural quantities reflect to some extent the buriedness of residues and the number of residue-residue contacts. PC2 and PC3 were more difficult to interpret. Since designed entropy and B factors loaded strongly on both but with two different combinations of signs, we concluded that the most parsimonious interpretation was to consider PC2 as a component representing sites with high designed entropy and high spatial fluctuations (as measured by B factors) and PC3 representing sites with high designed entropy and low spatial fluctuations. Using these interpretations, our PC regression analysis suggested that of all the structural quantities considered here, residue buriedness/packing was the best predictor of evolutionary variation. Designed entropy was a useful predictor as well, but it tended to perform better at sites with low spatial fluctuations.

For a second PC analysis, we included the predictor CS RMSF, which therefore restricted the data set to include only six proteins (see Table 2). This analysis, which retained sequence entropy as the response variable, yielded comparable results to the first PC analysis. The main differences occurred in PC2 and PC3, where CS RMSF generally loaded in the opposite direction of B factor, and either in the same (PC2) or the opposite (PC3) direction of designed entropy (Figure S1).

Finally, we redid the two PC analyses described above, but instead with $\omega$ as the response variable (Figures S2 and S3). Again, these results were largely comparable to results from PC analyses with sequence entropy as the response.

\section{Discussion}

We have carried out a comprehensive analysis of the extent to which different structural quantities predict sequence evolutionary variation in nine viral proteins. We found that measures of buriedness and local packing generally performed better than did measures of structural flexibility. Further, the former measures also performed better than a computational protein-design approach that employed a sophisticated all-atom force field to determine allowed amino-acid distributions at each site. Finally, there was no difference in predictive power between structural quantities obtained from averaging structural quantities over $15 \mathrm{~ns}$ of MD simulations versus taking the same quantities from individual crystal structures.

Our results are broadly in agreement with recent work by Echave and collaborators (Yeh et al, 2014b, Huang et al, 2014). These authors found that RSA and CN showed comparable correlation strengths with evolutionary sequence variation (Yeh et al, 2014b). Further, they demonstrated that the 
bioRxiv preprint doi: https://doi.org/10.1101/004481; this version posted July 21, 2014. The copyright holder for this preprint (which was

observed relationship between evolutionary variation and residue-residue contacts was not consistent with a flexibility model that puts evolutionary variability in proportion to structural flexibility (Huang et al, 2014). Instead, a mechanistic stress model, in which amino-acid substitutions cause physical stress in proportion to the number of residue-residue contacts affected, could explain all the observed data (Huang et al, 2014).

The correlation strengths we observed were consistently lower than those observed previously (Jackson et al, 2013, Yeh et al, 2014b). We believe that this result was due to our choice of analyzing viral proteins instead of the cellular proteins or enzymes used in prior works. First, while viral sequences are abundant, their alignments may not be as diverged as alignments that can be obtained for sequences from cellular organisms. For example, our influenza sequences spanned only approximately one decade. Despite the high mutation rates observed in RNA viruses, the evolutionary variation that can accumulate over this time span is limited. This relatively lower evolutionary divergence makes resolving differences between more and less conserved sites much more difficult. Second, many viral proteins experience a substantial amount of selection pressure to evade host immune responses. The resulting positive selection on viral sequences may mask evolutionary constraints imposed by structure. For example, influenza hemagglutinin displays positive selection throughout the entire sequence, regardless of the extent of residue burial (Meyer and Wilke, 2013, Meyer et al, 2013, Suzuki, 2006, Bush et al, 1999). However, the results we obtained here for viral proteins are broadly consistent with the results obtained earlier for cellular proteins (Dokholyan and Shakhnovich, 2001, Franzosa and Xia, 2009, Jackson et al, 2013, Yeh et al, 2014b), indicating that viral proteins evolve under many of the same biophysical selection pressures that cellular proteins experience.

We have found here that correlations between sequence entropy and structural quantities were consistently higher than correlations between the evolutionary-rate ratio $\omega$ and structural quantities. Surprisingly, in a recent study on cellular proteins, Yeh et al (2014a) found that entropy performed worse than quantities assessing substitution rates. One possible explanation for this discrepancy is again our choice of viral sequences. Our sequence alignments almost certainly contained some polymorphisms, whereas the sequences of Yeh et al (2014a) likely did not. It is known that polymorphisms may diminish the reliability of $\omega$ estimates (Kryazhimskiy and Plotkin, 2008). While the effect of polymorphisms on sequence entropy is not known, it seems plausible that entropy would be less sensitive to them than $\omega$ is. Alternatively, since viral proteins frequently experience positive selection, rate estimates may be confounded by this selection pressure and thus less reflective of constraints imposed by protein structure. By contrast, even under positive selection amino-acid distributions at sites would have to be consistent with the constraints imposed by the protein structure, and entropy would remain sensitive to these constraints.

We found that simple measures of buriedness or packing density, such as RSA or CN, were better predictors of evolutionary variation than was sequence variability predicted from computational protein design. In other words, simple quantities that can be obtained trivially from PDB structures performed better than a sophisticated protein-design strategy that makes use of an all-atom energy function and requires thousands of CPU-hours to complete. This result highlights that, even though computational protein design has yielded impressive results in specific cases (Kuhlman et al, 2003, Röthlisberger et al, 2008; Fleishman et al, 2011), this approach remains limited in its ability to predict evolutionary variation. Similarly, we have previously found that flexible backbone design with Rosetta produced designs whose surface and core were too similar (Jackson et al, 2013). We attributed this discrepancy to either the solvation model or the model of backbone flexibility we used (Backrub, see Smith and Kortemme 2008). The results we found here suggest that the model of backbone flexibility may indeed be the cause of at least some of the discrepancies between predicted and observed site variability. In particular, in our $\mathrm{PC}$ regression analysis, the component in which designed entropy loaded opposite to B factor and MD RMSF generally had the second-highest predictive power for evolutionary variability, after the component representing buriedness/packing density. In sum, designed entropy was a better predictor for evolutionary sequence variability for sites with less structural flexibility compared to sites with more flexibility.

Even though RSA and CN remain the best currently known predictors of evolutionary variation, neither quantity has particularly high predictive power. One reason why predictive power may be low is that neither quantity accounts for correlated substitutions at interacting sites. Yet such correlated substitutions happen regularly. For example, covariation among sites encodes information about residueresidue contacts and 3D structure (Halabi et al, 2009, Burger and van Nimwegen, 2010, Marks et al, 2011, Jones et al, 2014), and evolutionary models that incorporate residue-residue interactions tend to perform better than models that do not (Rodrigue et al, 2005, Bordner and Mittelmann, 2014). An improved predictor of evolutionary variation would have to correctly predict this covariation from structure. In principle, computational protein design, which takes into consideration the atom-level details of the 
bioRxiv preprint doi: https://doi.org/10.1101/004481; this version posted July 21,2014 . The copyright holder for this preprint (which was

protein structure, should properly reproduce covariation among sites. However, a recent analysis showed that there are significant limitations to the covariation that is predicted (Ollikainen and Kortemme, 2013). In addition, covariation in designed proteins is quite sensitive to the type of backbone variation modeled during design, and improved models of backbone flexibility may be required for improved prediction of covariation among sites (Ollikainen and Kortemme, 2013).

\section{Acknowledgments}

This work was supported in part by NIH grant R01 GM088344, DTRA grant HDTRA1-12-C-0007, ARO grant W911NF-12-1-0390, and the BEACON Center for the Study of Evolution in Action (NSF Cooperative Agreement DBI-0939454). The Texas Advanced Computing Center at UT Austin provided high-performance computing resources.

\section{References}

Berens P (2009) CircStat: a MATLAB toolbox for circular statistics. J Stat Software 31:1-21

Bloom JD, Drummond DA, Arnold FH, Wilke CO (2006) Structural determinants of the rate of protein evolution in yeast. Mol Biol Evol 23:1751-1761

Bordner AJ, Mittelmann HD (2014) A new formulation of protein evolutionary models that account for structural constraints. Mol Biol Evol 31:736-749

Burger L, van Nimwegen E (2010) Disentangling direct from indirect co-evolution of residues in protein alignments. PLoS Comput Biol 6:e1000,633

Bush RM, Bender CA, Subbarao K, Cox NJ, Fitch WM (1999) Predicting the evolution of human influenza A. Science 286:1921-1925

Dean AM, Neuhauser C, Grenier E, Golding GB (2002) The pattern of amino acid replacements in $\alpha / \beta$-barrels. Mol Biol Evol 19:1846-1864

Dokholyan NV, Shakhnovich EI (2001) Understanding hierarchical protein evolution from first principles. J Mol Biol 312:289-307

Drummond DA, Raval A, Wilke CO (2006) A single determinant dominates the rate of yeast protein evolution. Mol Biol Evol 23:327-337

Echave J, Fernández FM (2010) A perturbative view of protein structural variation. Proteins: Structure, Function, and Bioinformatics 78:173-180

Fleishman SJ, Whitehead TA, Ekiert DC, Dreyfus C, Corn JE, Strauch EM, Wilson IA, Baker D (2011) Computational design of proteins targeting the conserved stem region of influenza hemagglutinin. Science 332:816-821

Franzosa EA, Xia Y (2009) Structural determinants of protein evolution are context-sensitive at the residue level. Mol Biol Evol 26:2387-2395

Franzosa EA, Xia Y (2012) Independent effects of protein core size and expression on residue-level structure-evolution relationships. PLoS ONE 7:e46,602

Goldman N, Yang Z (1994) A codon-based model of nucleotide substitution for protein-coding DNA sequences. Mol Biol Evol 11:725-736

Goldman N, Thorne JL, Jones DT (1998) Assessing the impact of secondary structure and solvent accessibility on protein evolution. Genetics 149:445-458

Grant BJ, Rodrigues APC, ElSawy KM, McCammon AJ, Caves LSD (2006) Bio3D: an R package for the comparative analysis of protein structures. Bioinformatics 22:2695-2696

Halabi N, Rivoire O, Leibler S, Ranganathan R (2009) Protein sectors: Evolutionary units of threedimensional structure. Cell 138:774-786

Halle B (2002) Flexibility and packing in proteins. Proc Natl Acad Sci USA 99:1274-1279

Huang TT, del Valle Marcos ML, Hwang JK, Echave J (2014) A mechanistic stress model of protein evolution accounts for site-specific evolutionary rates and their relationship with packing density and flexibility. BMC Evol Biol 14:78

Jackson EL, Ollikainen N, III AWC, Kortemme T, Wilke CO (2013) Amino-acid site variability among natural and designed proteins. PeerJ 1:e211

Jones DT, Buchan DWA, Cozzetto D, Pontil M (2014) PSICOV: precise structural contact prediction using sparse inverse covariance estimation on large multiple sequence alignments. Mol Biol Evol 31:736749 
bioRxiv preprint doi: https://doi.org/10.1101/004481; this version posted July 21, 2014. The copyright holder for this preprint (which was

Jorgensen WL, Chandrasekhar J, Madura JD, Impey RW, Klein ML (1983) Comparison of simple potential functions for simulating liquid water. The Journal of Chemical Physics 79(2):926-935, DOI 10.1063/1.445869, URL http://scitation.aip.org/content/aip/journal/jcp/79/2/10.1063/1. 445869

Kabsch W, Sander C (1983) Dictionary of protein secondary structure: pattern recognition of hydrogenbonded and geometrical features. Biopolymers 22:2577-2637

Karplus M, McCammon A (2002) Molecular dynamics simulations of biomolecules. Nature Struct Biol 9:646-652

Katoh K, Misawa K, Kuma KI, Miyata T (2002) MAFFT: a novel method for rapid multiple sequence alignment based on fast Fourier transform. Nucl Acids Res 30:3059-3066

Katoh K, Kuma KI, Toh H, Miyata T (2005) MAFFT version 5: improvement in accuracy of multiple sequence alignment. Nucl Acids Res 33:511-518

Kosakovsky Pond SL, Frost SDW, Muse SV (2005) HyPhy: hypothesis testing using phylogenetics. Bioinformatics 21:676-679

Kryazhimskiy S, Plotkin JB (2008) The population genetics of dN/dS. PLoS Genet 4:e1000,304

Kuhlman B, Dantas G, Ireton G, Gabriele V, Stoddard B (2003) Design of a novel globular protein fold with atomic-level accuracy. Science 302:1364-1368

Leaver-Fay A, Tyka M, Lewis SM, Lange OF, Thompson J, Jacak R, Kaufman K, Renfrew DP, Smith CA, Sheffler W, Davis IW, Cooper S, Treuille A, Mandell DJ, Richter F, Ban YEA, Fleishman SJ, Corn JE, Kim DE, Lyskov S, Berrondo M, Mentzer S, Popović Z, Havranek JJ, Karanicolas J, Das R, Meiler J, Kortemme T, Gray JJ, Kuhlman B, Baker D, Bradley P (2011) ROSETTA3: an object-oriented software suite for the simulation and design of macromolecules. Methods in Enzymology 487:545-574

Liao H, Yeh W, Chiang D, Jernigan RL, Lustig B (2005) Protein sequence entropy is closely related to packing density and hydrophobicity. PEDS 18:59-64

Liberles DA, Teichmann SA, Bahar I, Bastolla U, Bloom J, BornbergBauer E, Colwell LJ, de Koning APJ, Dokholyan NV, Echave J, Elofsson A, Gerloff DL, Goldstein RA, Grahnen JA, Holder MT, Lakner C, Lartillot N, Lovell SC, Naylor G, Perica T, Pollock DD, Pupko T, Regan L, Roger A, Rubinstein N, Shakhnovich E, Sjölander K, Sunyaev S, Teufel AI, Thorne JL, Thornton JW, Weinreich DM, Whelan S (2012) The interface of protein structure, protein biophysics, and molecular evolution. Protein Sci 21:769-785

Liu Y, Bahar I (2012) Sequence evolution correlates with structural dynamics. Mol Biol Evol 29:22532263

Maguida S, Fernandez-Albertia S, Echave J (2008) Evolutionary conservation of protein vibrational dynamics. Gene 422:7-13

Marks DS, Colwell LJ, Sheridan R, Hopf TA, Pagnani A, Zecchina R, Sander C (2011) Protein 3D structure computed from evolutionary sequence variation. PLoS ONE 6:e28,766

Marsh JA, Teichmann SA (2014) Parallel dynamics and evolution: Protein conformational fluctuations and assembly reflect evolutionary changes in sequence and structure. BioEssays 36:209-218

Meyer AG, Wilke CO (2013) Integrating sequence variation and protein structure to identify sites under selection. Mol Biol Evol 30:36-44

Meyer AG, Dawson ET, Wilke CO (2013) Cross-species comparison of site-specific evolutionary-rate variation in influenza haemagglutinin. Phil Trans R Soc B 368:20120,334

Mirny LA, Shakhnovich EI (1999) Universally conserved positions in protein folds: reading evolutionary signals about stability, folding kinetics and function. J Mol Biol 291:177-196

Nevin Gerek Z, Kumar S, Banu Ozkan S (2013) Structural dynamics flexibility informs function and evolution at a proteome scale. Evolutionary Applications 6:423-433

Ollikainen N, Kortemme T (2013) Computational protein design quantifies structural constraints on amino acid covariation. PLoS Comput Biol 9:e1003,313

Overington J, Donnelly D, Johnson MS, Sali A, Blundell TL (1992) Environment-specific amino acid substitution tables: tertiary templates and prediction of protein folds. Protein Sci 1:216-226

Ramsey DC, Scherrer MP, Zhou T, Wilke CO (2011) The relationship between relative solvent accessibility and evolutionary rate in protein evolution. Genetics 188:479-488

Rodrigue N, Lartillot N, Bryant D, Philippe H (2005) Site interdependence attributed to tertiary structure in amino acid sequence evolution. Gene 347:207-217

Röthlisberger D, Khersonsky O, Wollacott AM, Jiang L, DeChancie J, Betker J, Gallaher JL, Althoff EA, Zanghellini A, Dym O, Albeck S, Houk KN, Tawfik DS, Baker D (2008) Kemp elimination catalysts by computational enzyme design. Nature 453:190-195 
bioRxiv preprint doi: https://doi.org/10.1101/004481; this version posted July 21, 2014. The copyright holder for this preprint (which was

Ryckaert JP, Ciccotti G, Berendsen HJC (1977) Numerical integration of the cartesian equations of motion of a system with constraints: molecular dynamics of n-alkanes. J Comp Phys 23:327-341

Salomon-Ferrer R, Götz AW, Poole D, Le Grand S, Walker RC (2013) Routine microsecond molecular dynamics simulations with AMBER on GPUs. 2. Explicit Solvent Particle Mesh Ewald. J Chem Theory Comput 9:3878-3888

Sanejouand YH (2013) Elastic network models: theoretical and empirical foundations. Methods Mol Biol 924:601-616

Scherrer MP, Meyer AG, Wilke CO (2012) Modeling coding-sequence evolution within the context of residue solvent accessibility. BMC Evol Biol Submitted

Shih CH, Chang CM, Lin YS, Lo W, Hwang JK (2012) Evolutionary information hidden in a single protein structure. Proteins: Structure, Function, and Bioinformatics 80:1647-1657

Smith CA, Kortemme T (2008) Backrub-like backbone simulation recapitulates natural protein conformational variability and improves mutant side-chain prediction. J Mol Biol 380:742-756

Spielman SJ, Wilke CO (2013) Membrane environment imposes unique selection pressures on transmembrane domains of G protein-coupled receptors. J Mol Evol 76:172-182

Stamatakis A (2006) RAxML-VI-HPC: maximum likelihood-based phylogenetic analyses with thousands of taxa and mixed models. Bioinformatics 22:2688-2690

Stone EA, Sidow A (2007) Constructing a meaningful evolutionary average at the phylogenetic center of mass. BMC Bioinformatics 8:222

Suzuki Y (2006) Natural selection on the influenza virus genome. Mol Biol Evol 23:1902-1911

Tien MZ, Meyer AG, Sydykova DK, Spielman SJ, Wilke CO (2013) Maximum allowed solvent accessibilites of residues in proteins. PLOS ONE 8:e80,635

Wilke CO, Drummond DA (2010) Signatures of protein biophysics in coding sequence evolution. Cur Opin Struct Biol 20:385-389

Yang Z (2000) Maximum likelihood estimation on large phylogenies and analysis of adaptive evolution in human influenza virus A. J Mol Evol 51:423-432

Yeh SW, Huang TT, Liu JW, Yu SH, Shih CH, Hwang JK, Echave J (2014a) Local packing density is the main structural determinant of the rate of protein sequence evolution at site level. BioMed Research International 2014:572,409

Yeh SW, Liu JW, Yu SH, Shih CH, Hwang JK, Echave J (2014b) Site-specific structural constraints on protein sequence evolutionary divergence: Local packing density versus solvent exposure. Mol Biol Evol 31:135-139 
bioRxiv preprint doi: https://doi.org/10.1101/004481; this version posted July 21, 2014. The copyright holder for this preprint (which was

\section{Tables}

Table 1 PDB structures considered in this study.

\begin{tabular}{ccccc}
\hline Viral Protein & PDB ID & Chain & $\begin{array}{c}\text { Sequence } \\
\text { Length }\end{array}$ & $\begin{array}{c}\text { Number of } \\
\text { Sequences }\end{array}$ \\
\hline Hemagglutinin Precursor & 1RD8 & AB & 503 & 1039 \\
Dengue Protease Helicase & 2JLY & A & 451 & 2362 \\
West Nile Protease & 2FP7 & B & 147 & 237 \\
Japanese Encephalitis Helicase & 2Z83 & A & 426 & 145 \\
Hepatitis C Protease & 3GOL & A & 557 & 1021 \\
Rift Valley Fever Nucleoprotein & 3LYF & A & 244 & 95 \\
Crimean Congo Nucleocapsid & 4AQF & B & 474 & 69 \\
Marburg RNA Binding Domain & 4GHA & A & 122 & 42 \\
Influenza Nucleoprotein & 4IRY & A & 404 & 943 \\
\hline
\end{tabular}

Table 2 Availability of homologous crystal structures. Although most viral proteins have many PDB structures available, the sequence divergence among these structures is low. Therefore, when calculating RMSF from crystal structures, we considered only those proteins with at least five homologous structures at $5 \%$ pairwise sequence divergence (highlighted in bold).

\begin{tabular}{cccccc}
\hline Viral Protein & BLAST & \multicolumn{5}{c}{ Units $^{\mathrm{a}}$} & \multicolumn{4}{c}{ Une sequences } \\
\cline { 3 - 6 } & & all & $\geq 2 \%^{\mathrm{b}}$ & $\geq 5 \%^{\mathrm{b}}$ & $\geq 10 \%^{\mathrm{b}}$ \\
\hline Hemagglutinin Precursor & 63 & 17 & 10 & $\mathbf{9}$ & 7 \\
Dengue Protease Helicase & 31 & 13 & 7 & $\mathbf{7}$ & 7 \\
West Nile Protease & 21 & 16 & 10 & $\mathbf{7}$ & 6 \\
Japanese Encephalitis Helicase & 31 & 12 & 7 & $\mathbf{7}$ & 7 \\
Hepatitis C Protease & 302 & 33 & 10 & $\mathbf{5}$ & 4 \\
Rift Valley Fever Nucleoprotein & 95 & 9 & 5 & $\mathbf{5}$ & 5 \\
Crimean Congo Nucleocapsid & 7 & 4 & 3 & 2 & 2 \\
Marburg RNA Binding Domain & 63 & 9 & 5 & 3 & 3 \\
Influenza Nucleoprotein & 69 & 15 & 4 & 4 & 2 \\
\hline
\end{tabular}

a BLAST hits against all sequences in the PDB, excluding hits with $<35 \%$ sequence identity and $<90 \%$ alignment length

$\mathrm{b}$ Unique sequences at indicated minimum pairwise sequence divergence

Table 3 Correlations between quantities obtained from MD trajectories and from crystal structures. For each quantity and each protein, we calculated the Spearman correlation $\rho$ between the values obtained from MD time averages and the values obtained from viral protein crystal structures. Note that crystal structures for all nine proteins were used for RSA, $\mathrm{CN}$, and WCN calculations, but only the six proteins for which we had sufficient crystal structure variability were used for CS RMSF. We then calculated the minimum, maximum, mean, and standard deviation of these correlations.

\begin{tabular}{ccccc}
\hline Quantity & $\min \rho$ & $\max \rho$ & $\langle\rho\rangle$ & $\mathrm{SD}(\rho)$ \\
\hline RSA & 0.937 & 0.981 & 0.948 & 0.012 \\
CN & 0.964 & 0.993 & 0.976 & 0.008 \\
WCN & 0.973 & 0.991 & 0.984 & 0.006 \\
RMSF & 0.218 & 0.723 & 0.502 & 0.181 \\
\hline
\end{tabular}


bioRxiv preprint doi: https://doi.org/10.1101/004481; this version posted July 21, 2014. The copyright holder for this preprint (which was not certified by peer review) is the author/funder, who has granted bioRxiv a license to display the preprint in perpetuity. It is made available under aCC-BY-NC-ND 4.0 International license.

\section{Figures}

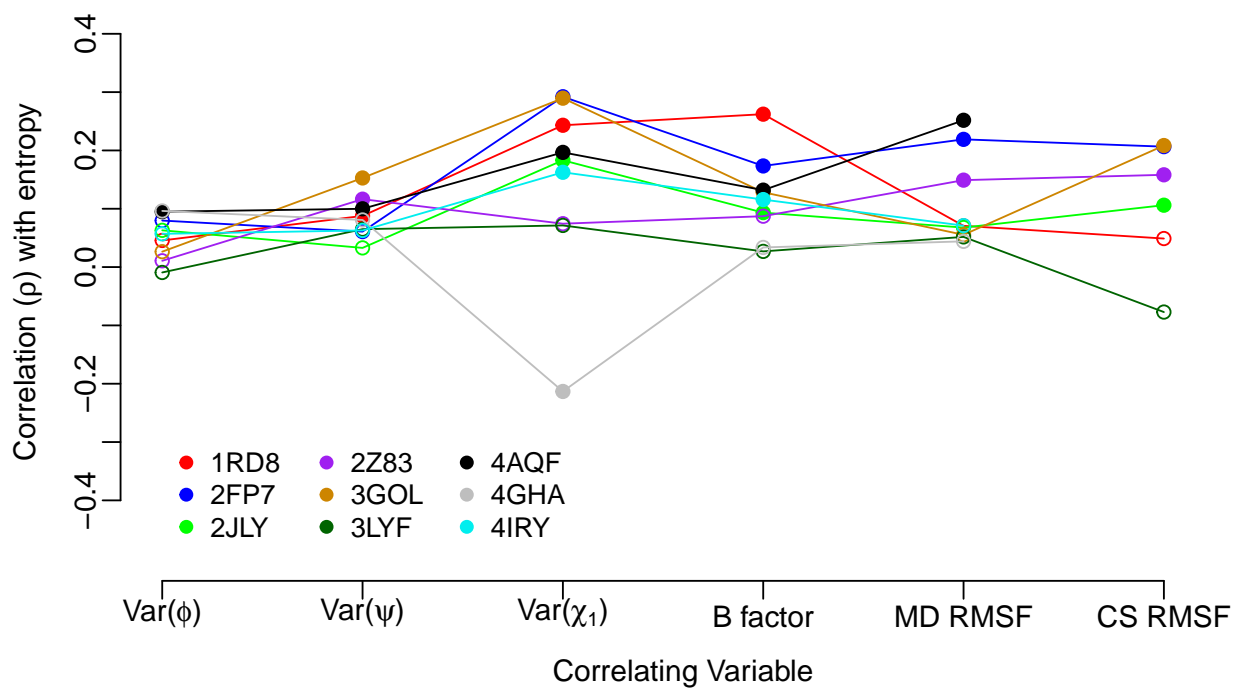

Fig. 1 Spearman correlation of sequence entropy with measures of structural variability. Each symbol represents one correlation coefficient for one protein structure. Significant correlations $(P<0.05)$ are shown as filled symbols, and insignificant correlations $(P \geq 0.05)$ are shown as open symbols. The quantities $\operatorname{Var}(\psi), \operatorname{Var}(\phi), \operatorname{Var}\left(\chi_{1}\right)$, and MD RMSF were obtained as time-averages over $15 \mathrm{~ns}$ of MD simulations. B factors were obtained from individual crystal structures. CS RMSF values were obtained from alignments of homologous crystal structures when available. Almost all structural measures of variability correlate weakly, but significantly, with sequence entropy.

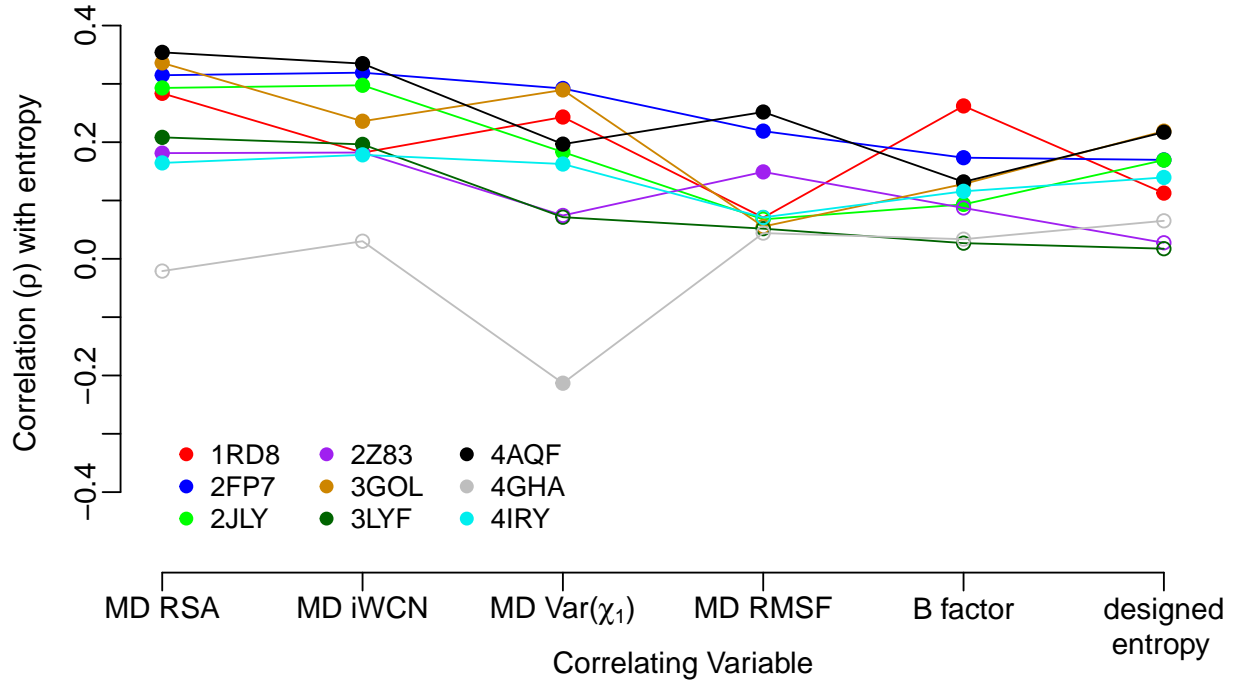

Fig. 2 Spearman correlation of sequence entropy with measures of buriedness, packing density, and structural flexibility, as well as with designed entropy. Each symbol represents one correlation coefficient for one protein structure. Significant correlations $(P<0.05)$ are shown as filled symbols, and insignificant correlations $(P \geq 0.05)$ are shown as open symbols. The quantities MD RSA, MD iWCN, MD $\operatorname{Var}\left(\chi_{1}\right)$, and MD RMSF were calculated as time-averages over $15 \mathrm{~ns}$ of MD simulations. B factors were obtained from crystal structures, and designed entropy was obtained from protein design in Rosetta. Compared to the measures of structural variability and to designed entropy, MD RSA and MD iWCN consistently show stronger correlations with sequence entropy. Note that results for MD iWCN are largely identical to those for MD iCN, so only MD iWCN was included here. 
A

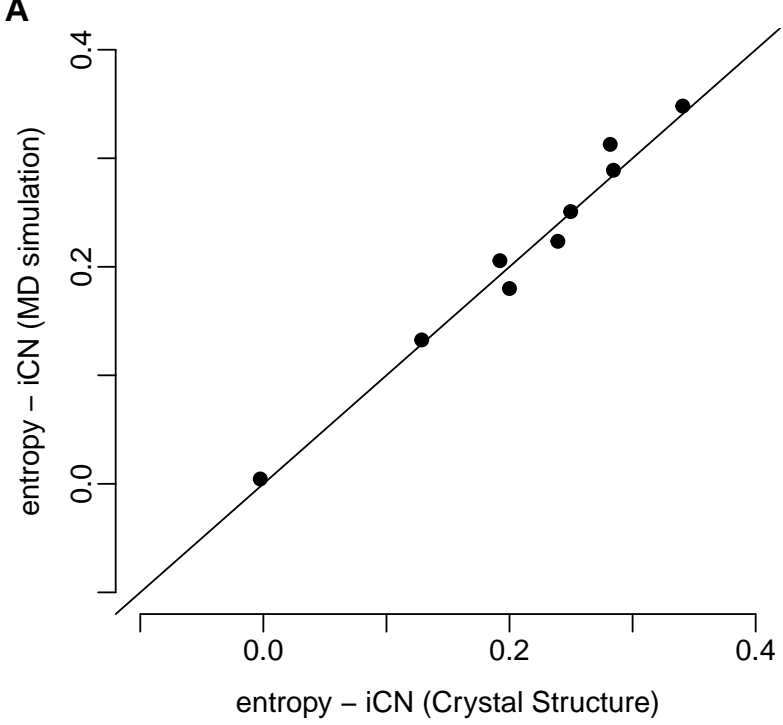

C

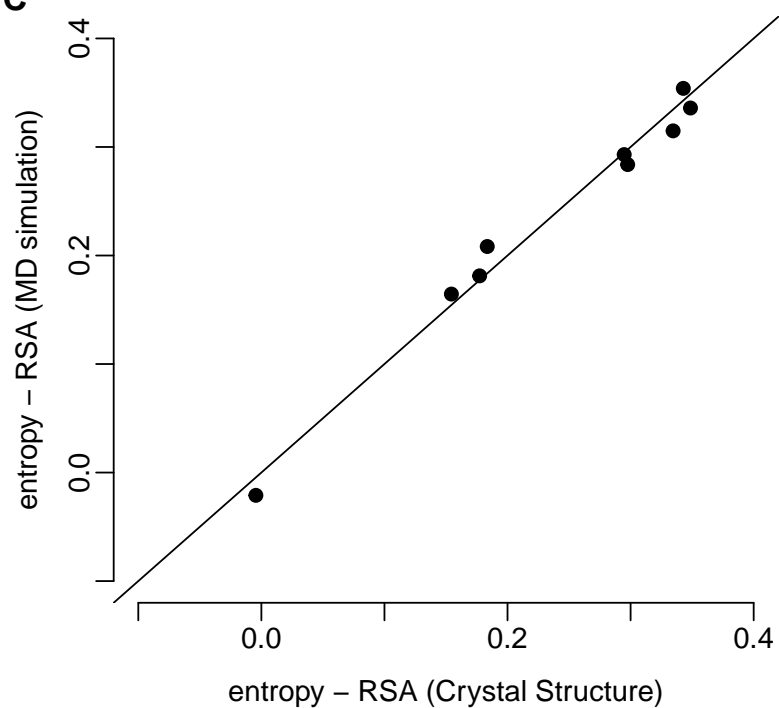

B

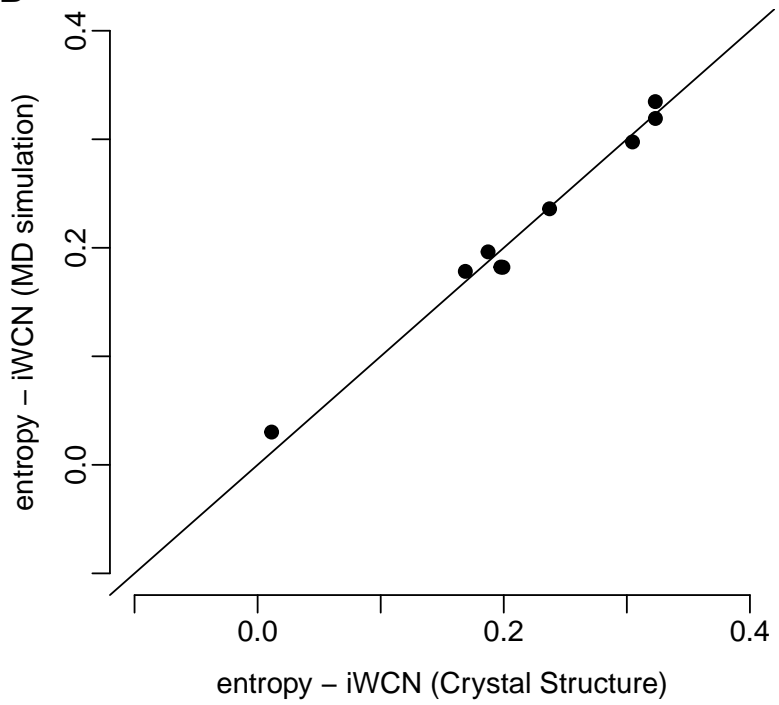

D

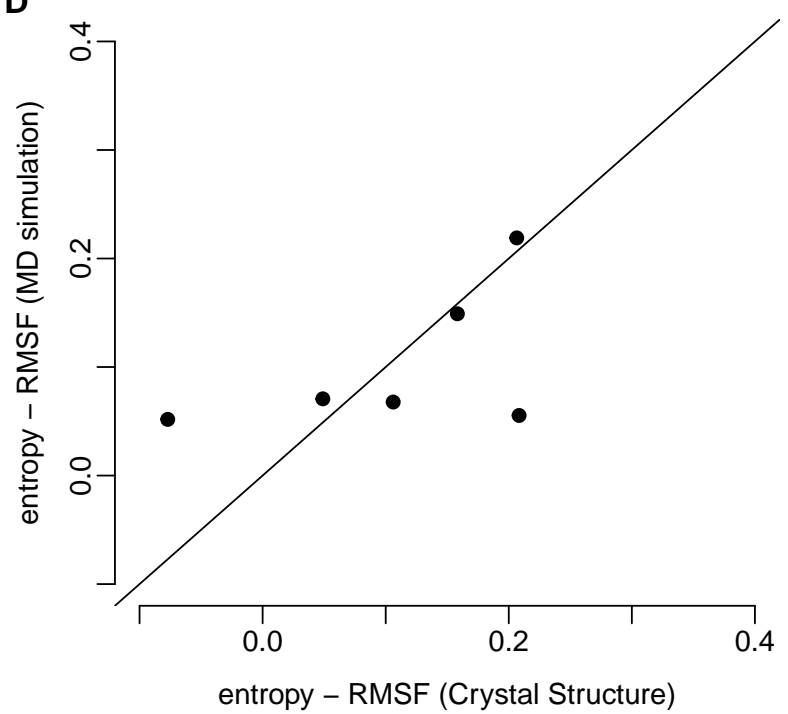

Fig. 3 Spearman correlations of sequence entropy with MD-derived and crystal-structure derived structural measures. The vertical axes in all plots represent the Spearman correlation of sequence entropy with one structural variable obtained from $15 n s$ of molecular dynamics (MD) simulations. The horizontal axes represent the Spearman's rank correlation coefficient of sequence entropy with the same structural variable as in the vertical axes but measured from protein crystal structures. Each dot represents one correlation coefficient for one protein structure. The quantities iCN, iWCN, and RSA have nearly identical predictive power for sequence entropy regardless of whether they are derived from MD simulations or from crystal structures. By contrast, MD RMSF yielded very different correlations than did CS RMSF. 
A

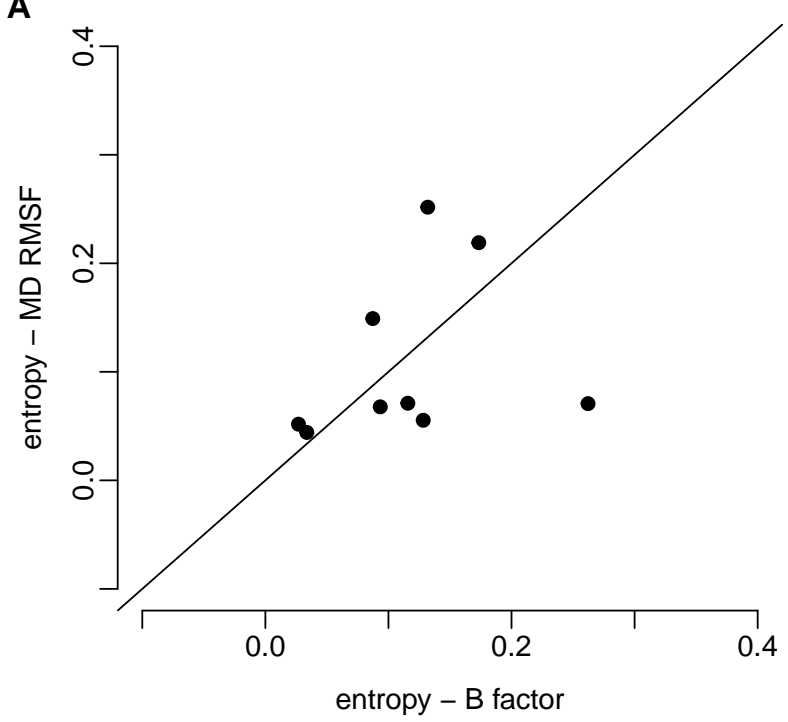

B

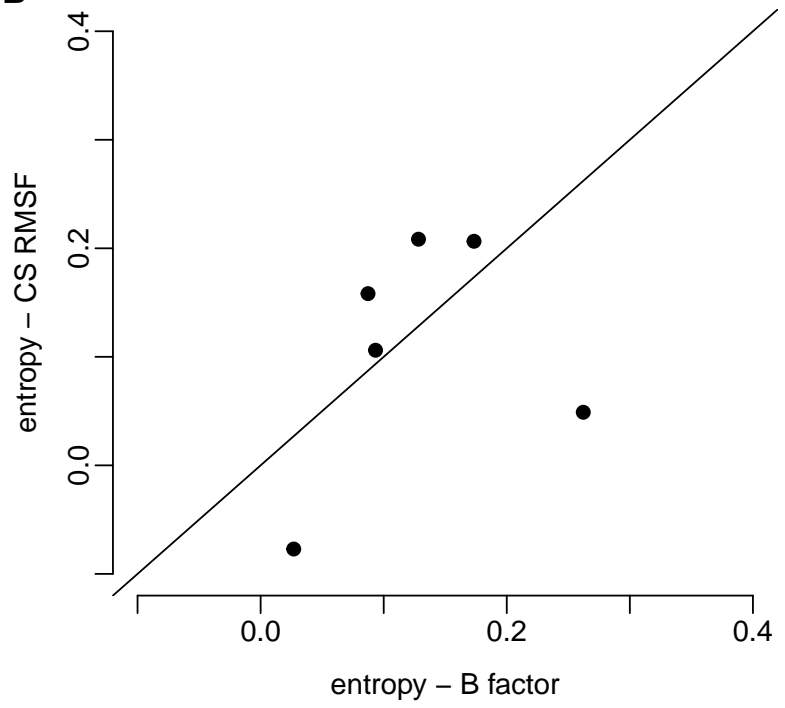

Fig. 4 Spearman correlations of sequence entropy with measures of structural variability. Vertical and horizontal axes represent Spearman correlations of the indicated quantities. Each dot represents one correlation coefficient for one protein structure. MD RMSF, CS RMSF, and B factors all explain different amounts of variance in sequence entropy for different proteins.

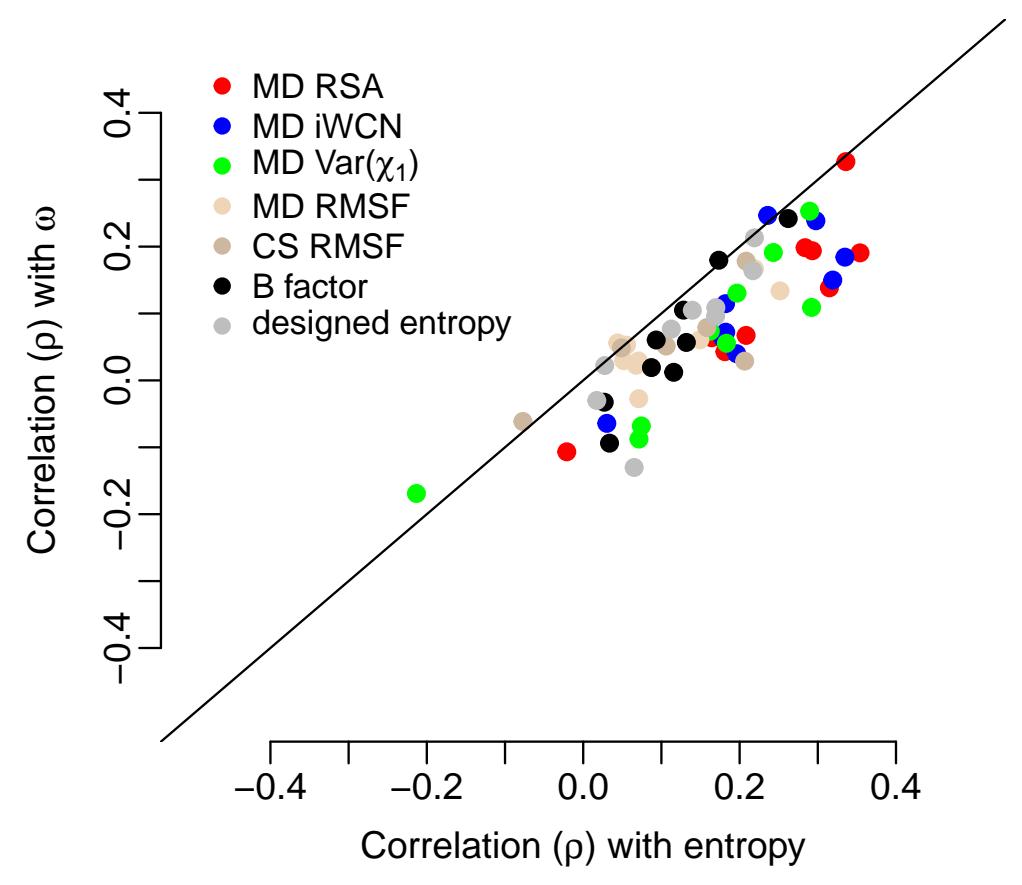

Fig. 5 Spearman correlations of structural quantities with sequence entropy and with the evolutionary rate ratio $\omega$. Nearly all points fall below the $x=y$ line, indicating that structural quantities generally predict as much as or more variation in sequence entropy than in $\omega$. 
bioRxiv preprint doi: https://doi.org/10.1101/004481; this version posted July 21, 2014. The copyright holder for this preprint (which was not certified by peer review) is the author/funder, who has granted bioRxiv a license to display the preprint in perpetuity. It is made available under aCC-BY-NC-ND 4.0 International license.
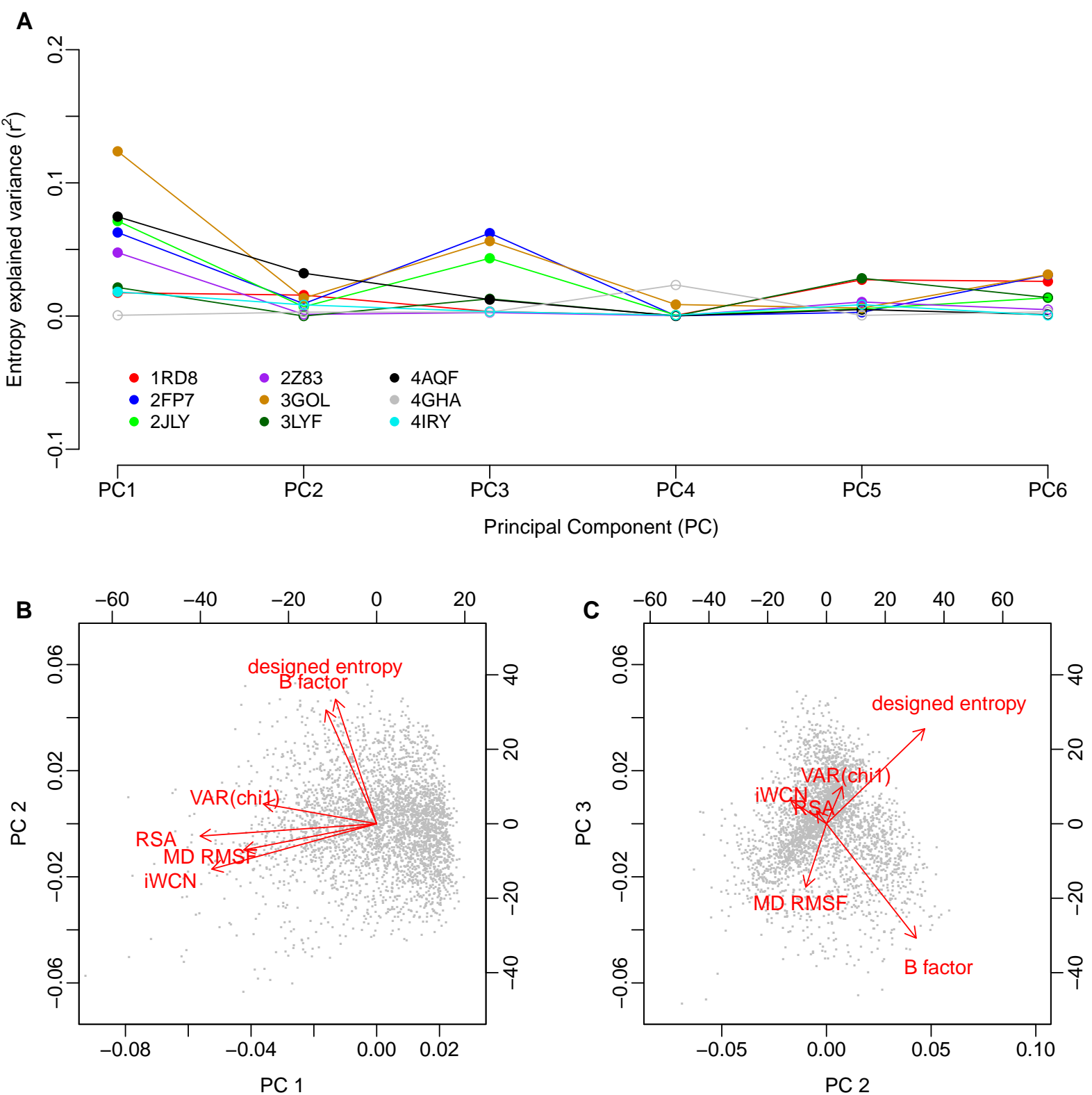

Fig. 6 Principal Component (PC) Regression of sequence entropy against the structural variables. (A) Variance in entropy explained by each principal component. For most proteins, PC1 and PC3 show the strongest correlations with sequence entropy. Significant correlations $(P<0.05)$ are shown as filled symbols, and insignificant correlations $(P \geq 0.05)$ are shown as open symbols. (B) and (C) Composition of the three leading components. Red arrows represent the loadings of each of the structural variables on the principal components; black dots represent the amino acid sites in the PC coordinate system. The variables RSA, iWCN, MD RMSF, and $\operatorname{Var}\left(\chi_{1}\right)$ load strongly on PC1 and weakly on PC2, while B factor and designed entropy load strongly on PC2 and weakly on PC1. Interestingly, B factor and designed entropy also load strongly on $\mathrm{PC} 3$, but in opposite directions. 


\section{Supplementary Figures}
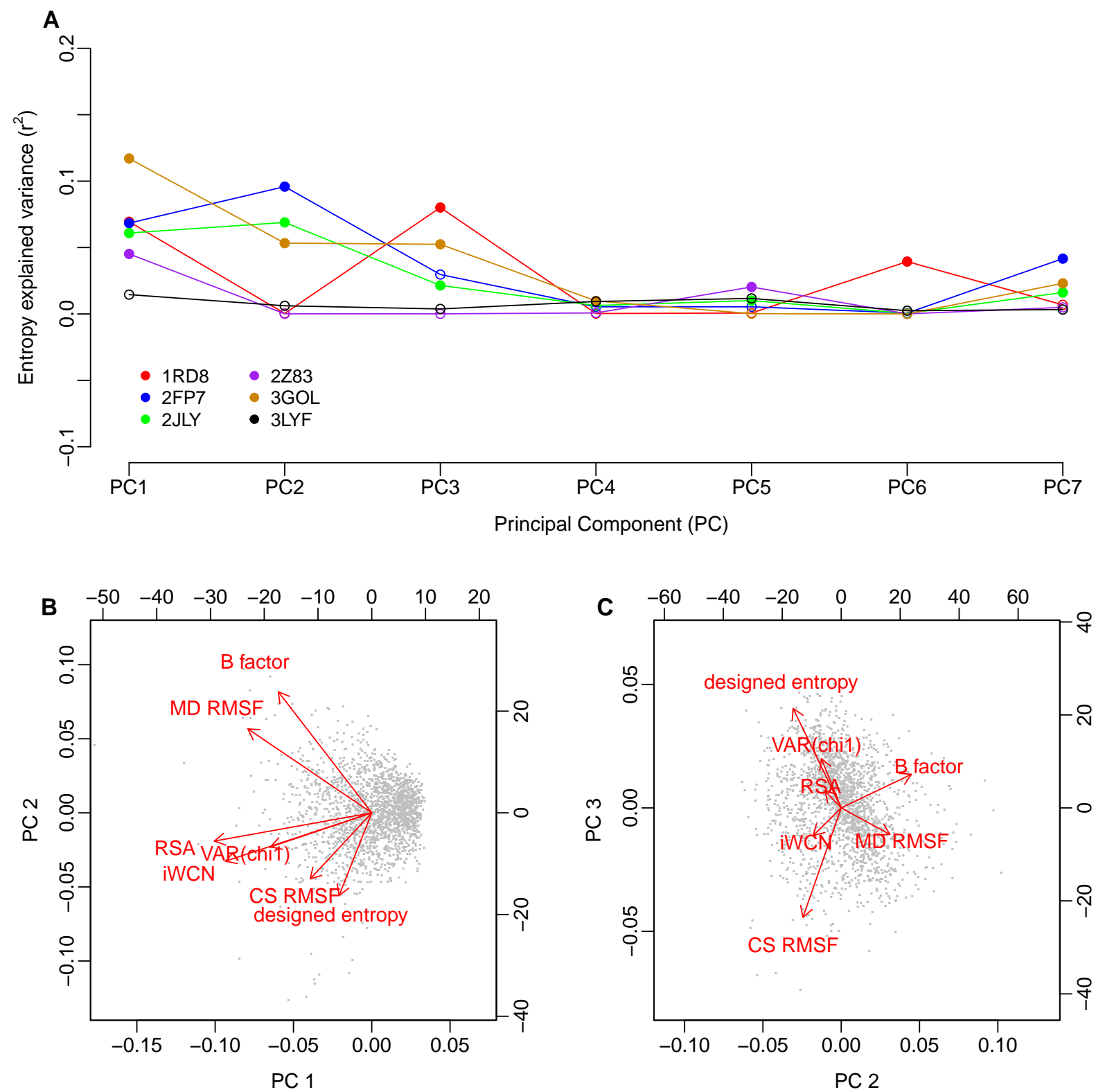

Fig. S1 Principal Component (PC) Regression of sequence entropy against the structural variables, including CS RMSF. (A) Variance in entropy explained by each principal component. For most proteins, PC1 and either PC2 or PC3 show the strongest correlations with sequence entropy. Significant correlations $(P<0.05)$ are shown as filled symbols, and insignificant correlations $(P \geq 0.05)$ are shown as open symbols. (B) and (C) Composition of the three leading components. Red arrows represent the loadings of each of the structural variables on the principal components; black dots represent the amino acid sites in the PC coordinate system. 

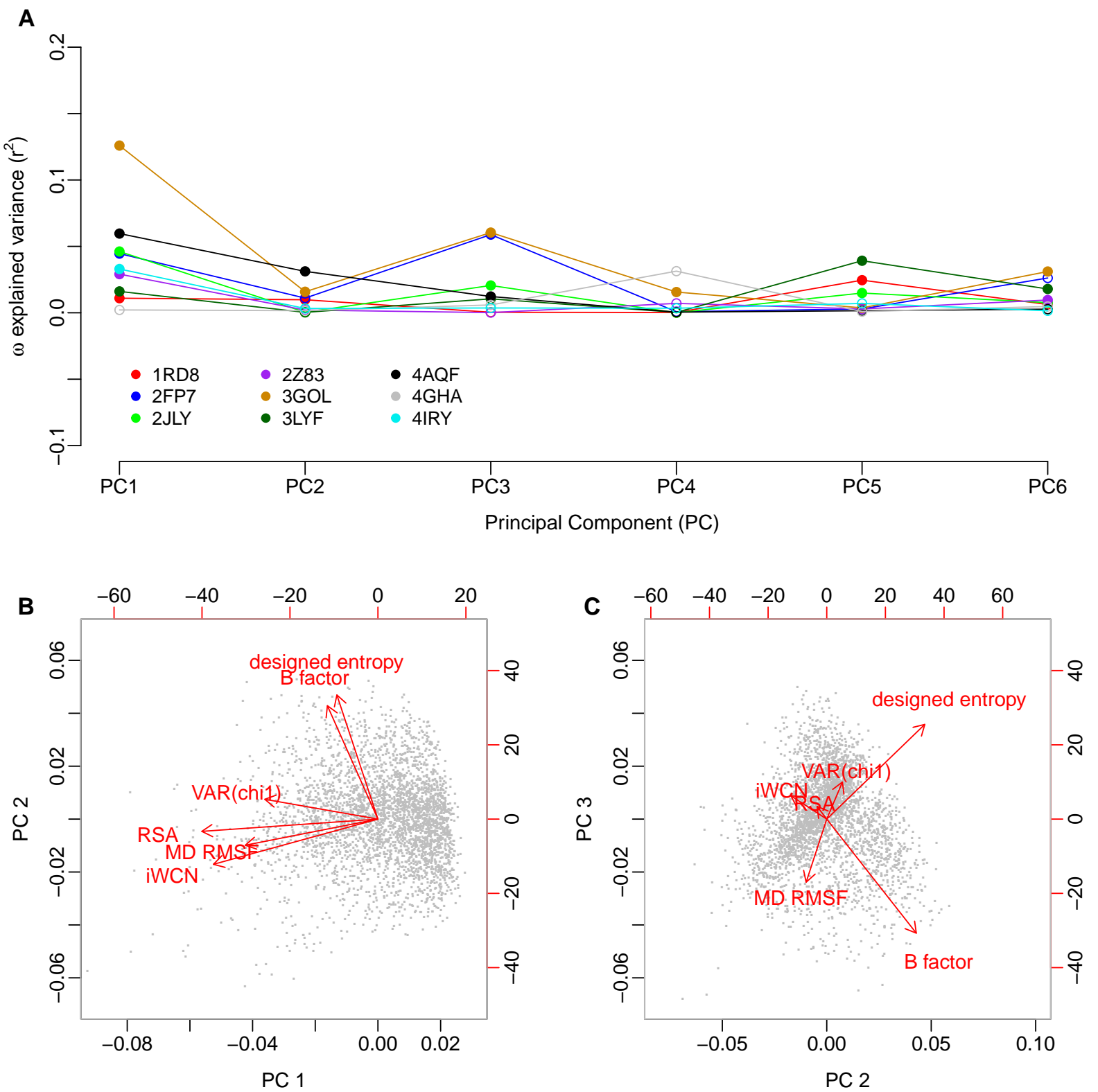

Fig. S2 Principal Component (PC) Regression of $\omega$ against the structural variables. (A) Variance in $\omega$ explained by each principal component. For most proteins, PC1 and PC3 show the strongest correlations with $\omega$. Significant correlations $(P<0.05)$ are shown as filled symbols, and insignificant correlations $(P \geq 0.05)$ are shown as open symbols. $(\mathbf{B})$ and $(\mathbf{C})$ Composition of the three leading components. Red arrows represent the loadings of each of the structural variables on the principal components; black dots represent the amino acid sites in the $\mathrm{PC}$ coordinate system. Note that parts $\mathrm{B}$ and $\mathrm{C}$ are identical to those shown in Figure 6. 

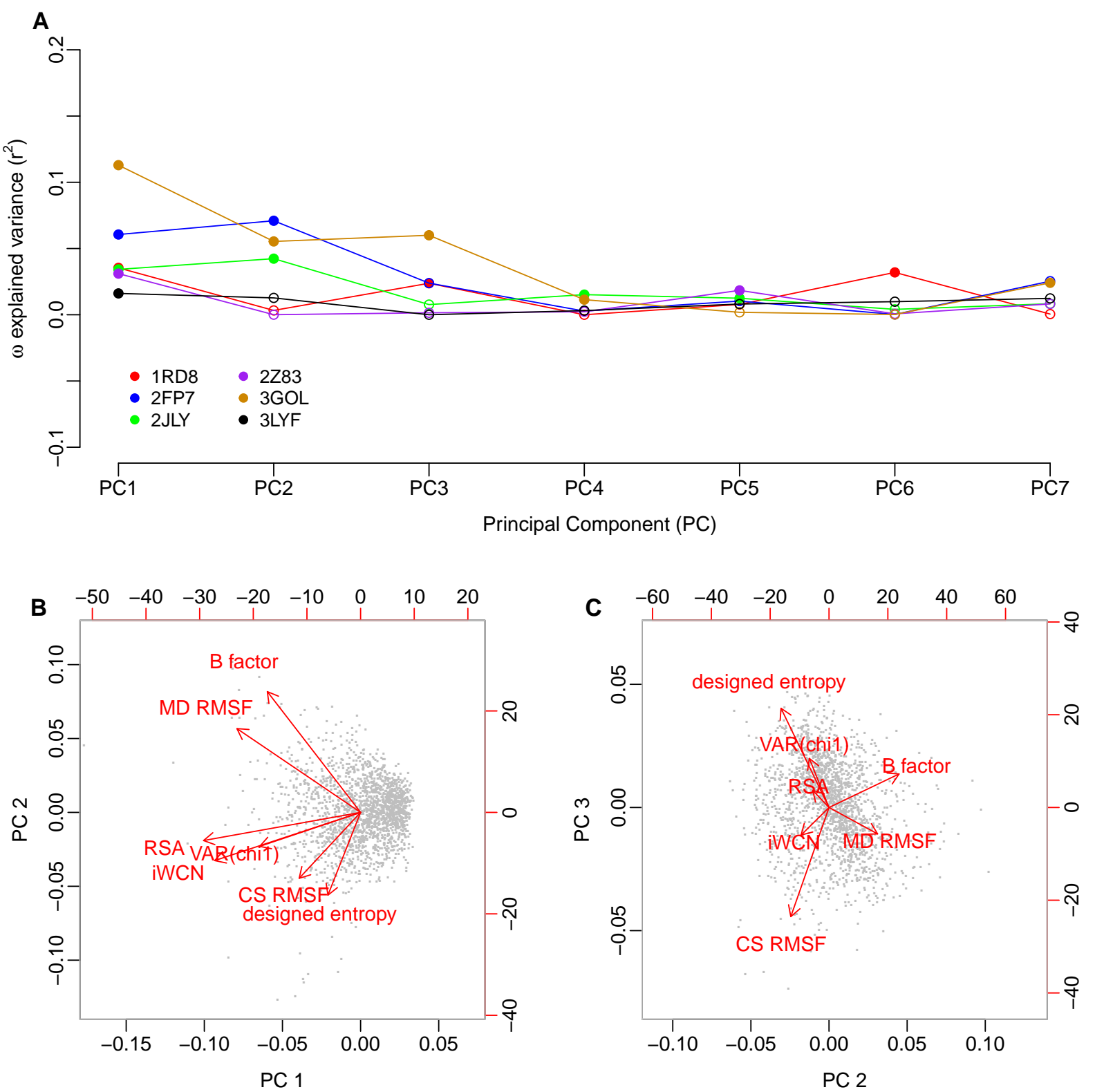

Fig. S3 Principal Component (PC) Regression of $\omega$ against the structural variables, including CS RMSF. (A) Variance in $\omega$ explained by each principal component. For most proteins, PC1 and either PC2 or PC3 show the strongest correlations with $\omega$. Significant correlations $(P<0.05)$ are shown as filled symbols, and insignificant correlations $(P \geq 0.05)$ are shown as open symbols. $(\mathbf{B})$ and $(\mathbf{C})$ Composition of the three leading components. Red arrows represent the loadings of each of the structural variables on the principal components; black dots represent the amino acid sites in the PC coordinate system. Note that parts B and C are identical to those shown in Figure S1. 